\title{
Editorial for the Journal of Textile Science and Engineering
}

\section{Xiaogang Chen*}

School of Materials, The University of Manchester, Manchester, UK

The textile industry is a "migrating" one with its outputs always being able to match the demand at the times of concern. From 1950's to early 1960 's, the textile production base migrated from North America and West Europe to Japan, and that sharply increased Japan's textile exports. From 1970s to 1980s, the textile production migrated again from Japan to the then so-called "Big Three"-Hong Kong, Taiwan, and South Korea, and then the Big Three dominated the textile exports. From the mid 1980's or so, the textile production base moved to the mainland China, the Southeast Asia nations, Sri Lanka and later on edged to South Asian and Latin American nations. Nowadays, there is a strong tendency that the textile production is going to the IndoChina nations and African countries. The reason for the industry's migration is closely associated to labour cost. Whilst the industrial base was migrated at different times, the latest technologies were absorbed into the textile industry, which much increased the textile output and stimulated the consumers' demand on textiles and clothing. For example, the current per capita consumption of textiles and clothing is more than doubled that of the 1950's, and the current world wide consumption of textiles and clothing is 5 times greater than that in the 1950 's. For the newly emerged technical textiles sector, the production and consumption demonstrated strong growth. The production of technical textiles in Europe increased 21\% between 1995 and 2012. The fibre consumption for technical textiles in the US witnessed an increase of $10 \%$ during the same period.Looking at these facts, one can only believe that the textile industry is far from a sun-set industry, but is a very vibrant one in the world economy.

The growth and progress in the textile industryhave been much dependent on the innovations in raw materials, textile manufacturing processes, and in end uses of textile materials and products. The manufacture of new types of fibres and the creative use of the natural fibres made the textile products and clothing more versatile in better meeting the performance and aesthetic requirements. The progress in textile processing led to high quality and high quantity of textiles and products. The technological change in the end uses of textiles has led to functional textiles that satisfy the engineering demands e.g. protection, reinforcing, and filtration.

Although textile technology has the longest history compared to others, many unknowns remain to be discovered for textile materials. This is particularly the case when new technology and new materials are absorbed into the textile engineering and manufacturing. The basic unit of textiles is fibre, and yarns are made from fibres and fabrics are made from yarns. As a result, the fabric, be it woven, knitted, braided, or even nonwoven, represents a very complicated type of material which is far from homogeneous and isotropic. The interactions between the fibres and the nature of the fibres both come into play to determine the performance of the textile fabric as an assembly. This nature of the textiles has made the mechanics and thermodynamics for textile assemblies more complex than their counterparts such as metal and plastics. Fortunately, the attempt to model the textiles has never ceased, as the relations among the material type, structure of fibre assembly, and assembly behavior need to be described accurately to guidethe development of textiles for the intended applications. This requirement is true for all types of textiles, but it would be more of the case for the advanced textile composites intended for the aerospace industry than for the textiles aimed for the domestic applications. The former cannot afford failure of materials during the service time whereas the failure of materials in the latter case would not be catastrophic.

Indeed, the advanced textile composites represent a highly research-intensive area. Not only the right fibre and matrix materials must be used, but the construction of the reinforcing textiles must be correct to achieve the required perform geometry, fibre orientation, lightweight, mechanical performance, thermal behavior and so on. The creation of required textile reinforcements requires, to say the least, the understanding of the property of the composites, the engineering of the architecture of the textile assembly, the availability of manufacturing process, the practical evaluation methods, and theoretical modeling.

Another example is the engineering of ballistic protective materials. The ballistic textiles require, among others, high level of protection against high velocity ballistic impact and low mass. For a given type of raw material, these two requirements on the textile assembly are contradictive because less material is expected to be used for higher level of protection. What is needed here is again correct understanding of the mechanism in which the textile assembly absorbs impact energy carried by the projectile. Such understanding will need to be translated to guide the design and engineering of the ballistic fabrics and assemblies. Textile mechanics and materials science has proven to important tools to solve the problems.

Textile technology probably is the only one that is able to convert linear materials into planar and spatial structures and materials, which offer unique properties and see many applications. Associated to the manufacturing and processing of textiles, pre- and post-treatment to textiles also add to and modify the performanceof textiles, that presents another dimension in textile science and engineering.

Now let us go back to the points made at the beginning of this editorial. The regions where textiles are produced may change and the materials and technologies involved in textile engineering may renew, but textile as an industry will be there for a long time. As such, innovations and advancements will have to be made to meet the ever-increasing demands from all application areas. Needless to say, the innovations and advancements depend on the creative work of all involved in the textile industry, including textile scientists, technologists, and engineers.

${ }^{*}$ Corresponding author: Xiaogang Chen, Executive Editor of the Journal of Textile Science \& Engineering, School of Materials, The University of Manchester, Manchester, UK, E-mail: xiaogang.chen@manchester.ac.uk

Received November 05, 2012; Accepted November 06, 2012; Published November 13, 2012

Citation: Chen X (2012) Editorial for the Journal of Textile Science and Engineering $\mathrm{J}$ Textile Sci Eng 2:e111. doi:10.4172/2165-8064.1000e111

Copyright: (c) 2012 Chen X. This is an open-access article distributed under the terms of the Creative Commons Attribution License, which permits unrestricted use, distribution, and reproduction in any medium, provided the original author and source are credited. 